\title{
Sharing of Psychological Nursing Experience of Patients with COVID-19
}

\author{
Juan Cheng ${ }^{*}$, Xiaoyan Zhang ${ }^{2}$ \\ ${ }^{1}$ Emergency Department, Xi'an Children's Hospital, Xi'an 710003, Shaanxi Province, China \\ ${ }^{2}$ Department of Internal Medicine, Shangnan County Hospital of Traditional Chinese Medicine, Shangluo 726300, \\ Shaanxi Province, China
}

\begin{abstract}
The author nursed suspected and confirmed cases of COVID-19 imported from abroad in a mobile hospital. During the front-line work in the fight against the epidemic in 2020, emergency response measures and corresponding clinical nursing experience have been accumulated for public health emergencies. This article summarized and shared the psychological nursing of patients with COVID-19 during the work of the author.
\end{abstract}

Key words: COVID-19; Psychological nursing

Publication date: May, 2021; Publication online: 31 May, 2021

*Corresponding author: Juan Cheng, JuanCheng@,sina.com

\section{Background}

Since December 2019, COVID-19 has occurred in Wuhan, Hubei Province. With the spread of the epidemic, such cases have been discovered in other regions of our country and many overseas countries. As an acute respiratory infectious disease, COVID-19 has been included in the category B infectious disease stipulated in the Law of the People's Republic of China on the Prevention and Treatment of Infectious Diseases and is managed as a category A infectious disease. By adopting a series of preventive control and medical treatment measures, the upward trend of the epidemic situation in our country has been contained to a certain extent. The epidemic situation has eased in most provinces, but the number of cases abroad is on the rise. "Imported cased prevention" is strictly enforced in our country. Family visits in the hospital and isolation wards are forbidden to implement closed management. The scope of patient activities is also restricted in the isolation wards, and those returning from overseas will be strictly screened and isolated. For patients with COVID-19, the most frightening thing is sometimes not the condition, but the patient's psychological state. A good psychological state is an important prerequisite for coping with COVID-19. Therefore, clinical nursing should be directed to the patient's psychology ${ }^{[1]}$. The author summarized and shared three points of experience in the psychological nursing of patients with suspected or confirmed COVID-19.

\section{Intervention of medical staff}

\subsection{Introduction to diseases and conditions}

Medical staff should take the initiative to introduce to patients the precautions for preventing infection with COVID-19, explain the knowledge of COVID-19 to patients, help patients understand the development of the disease, and help relieve patients' nervousness. It is worth noting that the accuracy and reliability of knowledge about COVID-19 should be guaranteed, so that patients have a clearer understanding of the severity of the epidemic, the possibility of infection and the protection methods. According to current case studies, most patients have a good prognosis, children and young people have mild symptoms, and severe cases are more common in the elderly and people with underlying diseases. Enabling patients to understand their own condition and tell them the relevant examination results can alleviate their anxiety.

\subsection{Psychological intervention behavior}

\subsubsection{Communication and quality nursing}


Nurses should pay special attention to patients with psychological anxiety or emotional disorders, visit the ward more, communicate with patients more, strengthen quality nursing services, understand the needs of patients, and actively provide help. During the development of clinical nursing for patients with COVID-19, the concept of humanistic nursing is introduced, avoiding prejudice, treating patients equally, understanding the frustration of patients, and encouraging patients to vent and receive isolation treatment. Patients imported from abroad have a long treatment experience and worry about their family members, which will inevitably cause anxiety and resistance. Therefore, the clinical nursing concept of "patient-centered", psychological nursing as the mainstay, and medical treatment as the supplement is advocated.

\subsection{2 "Ventilation" therapy}

Due to the particularity of the disease and the particularity of the layout of the isolation ward, and worried about the depression and anxiety of the patient, medical staff should do a good job of secondary protection, and take patients out of the ward in batches every day to ventilate and communicate (patients wear masks, and the distance between people is $>1 \mathrm{~m}$ ), which is called "ventilation therapy" and has been approved and praised by patients. It has effectively reduced the anxiety and fear of patients.

\subsubsection{Intervention of psychological counselors}

In the epidemic of COVID-19, effective psychological support and counseling for the public is of great significance for stabilizing the situation, resolving crises, stabilizing the society, uniting people's hearts, and establishing a positive and optimistic attitude of fighting against the disease ${ }^{[2]}$. Our mobile hospital has also set up a psychological counseling group to provide 24-hour online services. According to the needs of the patients, and with the consent of the patients, the psychological counselor can provide face-to-face psychological counseling or online psychological counseling with the patients.

\subsubsection{Environmental support and nursing}

In order to prevent respiratory droplet transmission, contact transmission and aerosol transmission, the isolation ward is managed in a closed manner, leaving only observation windows and transmission windows. In order to give patients a warm and comfortable home feeling, TVs and wireless networks are installed in the ward, "Welcome Home" letters are written to the patients, the hand-painted paintings of some warm medical staff and the pamphlet propagating disease knowledge are pasted on the inner wall, and the convenience of express delivery for patients is also provided.

\subsubsection{Family support}

This ward has established a basic family information form for quarantined patients. The information on this form can be used to contact the patients' family, mobilize their relatives and friends, mobilize their relatives and friends, encourage the family of the patients to communicate with the patients more, give the patients spiritual support actively, and build confidence in overcoming the disease ${ }^{[3]}$.

\section{Summary}

As COVID-19 is more clinically harmful and has a serious impact on the social development of our country, and the patients' isolation treatment is longer, clinical nursing work should pay more attention to the psychological nursing of patients while doing basic nursing work. In response to the psychological stress response of patients with COVID-19, medical workers should actively take various measures to eliminate the psychological pressure of the patients, reduce the patients' negative emotion, so as to promote the recovery of the disease ${ }^{[4]}$.

\section{References}

[1] Tang ZQ. Observation on the application effect of high-quality nursing intervention focusing on humanistic care on elderly patients with severe pneumonia [J]. Friends of Health, 2019, (15): 221.

[2] Li ZQ, Zhang ZM. Research on psychological support counseling model under the epidemic of COVID-19 [J]. Research and Practice of Health Care Medicine, 2020, 17(2): 8 .

[3] Xu QZ. The effect of health education on the burden and anxiety and depression of family caregivers with dementia at home [J]. Journal of Psychiatry, 2017, 30(2): 185-187.

[4] Bai JS, Wang Y, Yuan Y et al. Support nurses' psychological burden and management strategies when fighting against COVID-19 [J]. Qilu Nursing Journal, 2020, 26 (4): 9-11.

[5] COVID-19 Diagnosis and Treatment Plan (Trial Version 7) 